\title{
A Novel Robotic System for Inner Wall Derusting and Grinding of Pressure Pipelines
}

\author{
Xingji Du ${ }^{a}$, Defang Chen ${ }^{b}$, Wuhao Tan ${ }^{c}$, Zhangwei Ling ${ }^{d}$, Weican Guo ${ }^{\mathrm{e}}$, \\ Cunjian Miao ${ }^{f}$
}

Zhejiang Provincial Special Equipment Inspection and Research Institute, Hangzhou 310020, China

a497039204@qq.com, bglcdf@163.com, ctanwuhao@163.com, dlingzhangwei@163.com,

egwcndt@126.com, fmiaocunjian@163.com

Corresponding author: Zhangwei Ling

Keywords: In-pipe robot, Inner wall derusting and grinding, Pressure pipeline.

\begin{abstract}
Inner wall derusting and grinding of pressure pipelines are important work for their safety operation. However, up to date, this important work are still in difficulty, the corresponding methods are still very limited and cannot meet the requirements of practical industry applications. Therefore, the aim of this work is to investigate the practical inner wall derusting and grinding methods of the pressure pipelines. A novel robotic system for inner wall derusting and grinding of the pressure pipelines, which mainly includes a tracked mobile robot, a derusting and grinding module, and a control module, is developed. The tracked mobile robot is used to provide the driving force of the system movement. The derusting and grinding module is designed on the basis of a DC motor. The control module is used to control the motion state of the robot and the whole derusting and grinding process. Inner wall derusting and grinding experiments of the pressure pipelines with outer diameter of $159 \mathrm{~mm}$ are carried out. The research results demonstrate the effectiveness of the developed system.
\end{abstract}

\section{Introduction}

Pressure pipelines, which are important facilities for transporting oil, gas, water, and other fluids, widely exist in many industrial fields, such as petroleum, natural gas, metallurgy, chemical engineering, etc. During the long-term use of the pressure pipelines, the rusting and the scaling of their inner wall, which can easily lead to the local corrosion, the local overheat and hence cause the safety accidents, may arise. Therefore, derusting and grinding of their inner wall are important work for safety operation of the pressure pipelines.

Currently, the application of in-pipe robotic system for pressure pipeline inspection is considered as one of the most attractive solutions available and many research works concerning in-pipe robot have been reported [1-3]. Roh et al. [1] and Roman et al. [2] reviewed the development of the in-pipe inspection robotic system, respectively. Wang and $\mathrm{Gu}$ [4] presented a pipeline robot based on a bristle mechanism. The bristle mechanism with great flexibility can make the pipeline robot work in ill-constraint pipes. Qi et al. [5] overviewed two different robotic systems for in-pipe inspection of underground oil pipelines and mainly introduced the tracing and localization technology of in-pipe robot without tether cable.

Although great efforts of in-pipe robotic system have been made, few research works concerning inner wall derusting and grinding robot for pressure pipelines have been reported. Due to the small inner diameter and the complicated configuration of the practical pressure pipelines, the conventional derusting and grinding device cannot successfully run through inside the pipelines and cannot completely implement the corresponding inner wall derusting and grinding [6-9]. Therefore, inner wall derusting and grinding methods should be investigated.

This work aims to study the practical inner wall derusting and grinding methods of the pressure pipelines. A novel robotic system for the inner wall derusting and grinding of the pressure pipelines is 
developed. The details of the novel robotic system are presented. Inner wall derusting and grinding experiments of the pressure pipelines with outer diameter of $159 \mathrm{~mm}$ are also carried out.

\section{The novel robotic system}

The novel robotic system mainly includes three parts: (1) an in-pipe robot, (2) a derusting and grinding module and (3) a control module, as shown in Figure 1. The in-pipe robot is used to provide the driving force of the system movement. It can automatically move through inside the pressure pipelines with three caterpillar bands (driven by six motors) and the corresponding mechanical structures. The derusting and grinding module, which is connected with the in-pipe robot, is used for inner wall derusting and grinding of the pressure pipelines. The control module is used to control the motion state of the robot and the whole derusting and grinding process.

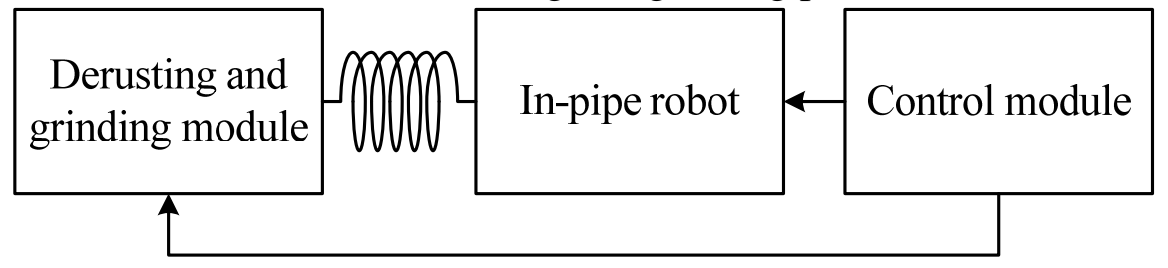

Fig. 1 Scheme of the novel robotic system

\subsection{The in-pipe robot}

Figure 2 shows the construction of the in-pipe robot, including the body frame, the corresponding mechanical parts installed in the body frame, and three caterpillar bands spaced at $120^{\circ}$. The three caterpillar bands are driven by six DC motors (each caterpillar band is equipped with two DC motors).

The in-pipe robot can automatically move through inside the pressure pipelines and can adapt to the pressure pipeline conditions, such as straight pipelines, welded joints, elbows, branches, and other special components. It uses the spring tensions (generated by the mechanical structures) to press the inside wall of the pressure pipelines and can also realize the adaption to pipe diameter and the adjustment of wall pressing force.

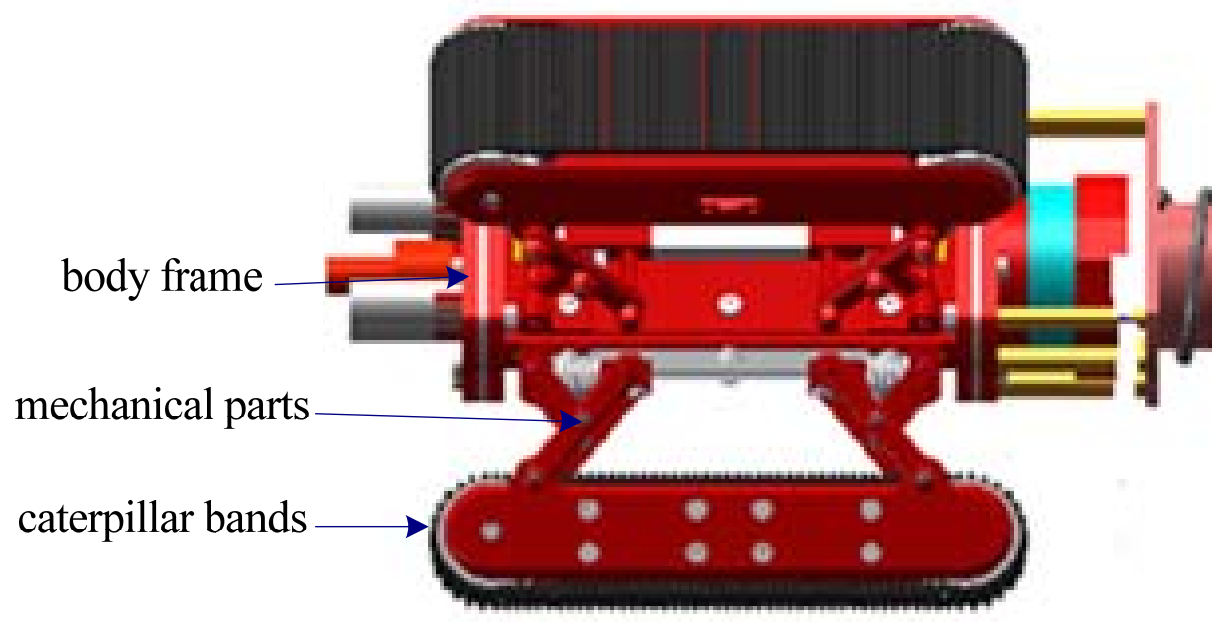

\subsection{The derusting and grinding module}

Fig. 2 Construction of the in-pipe robot

The derusting and grinding module mainly includes six parts: (1) the connection unit, (2) four rolling wheels, (3) a DC motor for derusting and grinding, (4) a metal shield, (5) the derusting and grinding components and (6) the spring mechanisms, as shown in Figure 3.

The connection unit is used to connect the in-pipe robot with the derusting and grinding module. Four rolling wheels are the movement components of the derusting and grinding module. The DC motor is used to control the rotational speed of the derusting and grinding components. The metal shield is used to protect the damage of the DC motor caused by the direct collision between the DC 
motor and the pipelines. The derusting and grinding components and the spring mechanism are used to implement the derusting and grinding process.

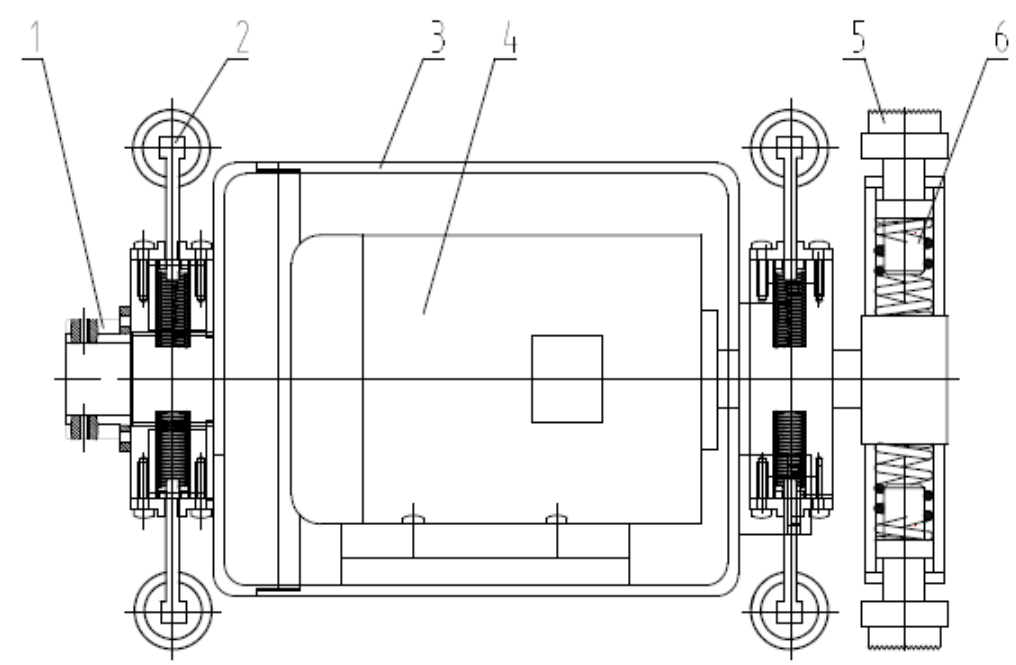

1. Connection unit, 2. Rolling wheel, 3. Metal shield, 4. DC motor for derusting and grinding,

5. Derusting and grinding components, 6. Spring mechanism

Fig. 3 Construction of the derusting and grinding module

\subsection{The control module}

The control module, which is designed on the basis of a micro controller, is used to control the motion state of the robot and the whole derusting and grinding process. During the movement process of the in-pipe robot, the control module send the real-time signals to control the six DC motors and hence to control the movement state of the three caterpillar bands (each caterpillar band is equipped with two DC motors). This procedure is implemented by the famous controller area network (CAN) bus and the corresponding communication protocol. Meanwhile, the rotational speed of the DC motor for derusting and grinding is adjusted by the control module.

\section{Discussions and conclusions}

Inner wall derusting and grinding of pressure pipelines are important work for their safety operation. However, up to date, this important work are still in difficulty, the corresponding methods are still very limited and cannot meet the requirements of practical industry applications.

This work aims to study the practical inner wall derusting and grinding methods of the pressure pipelines. A novel robotic system for the inner wall derusting and grinding of the pressure pipelines is developed. The main components of the novel robotic system including the in-pipe robot, the derusting and grinding module, and the control module, are presented. Inner wall derusting and grinding experiments of the pressure pipelines with outer diameter of $159 \mathrm{~mm}$ are carried out. The research results demonstrate the effectiveness of the developed system.

\section{Acknowledgements}

Corresponding author: Zhangwei Ling; E-mail: lingzhangwei@163.com.

This work was supported by the Science and Technique Plans of Zhejiang Province under Grant 2015 C33013.

\section{References}

[1] S. Roh and H. R. Choi. Differential-Drive In-Pipe Robot for Moving Inside Urban Gas Pipelines. IEEE Transactions on Robotics, 2005, 21(1), p. 1-17.

[2] H. T. Roman, B. A. Pellegrino, and W. R. Sigrist. Pipe Crawling Inspection Robots: An Overview. IEEE Transactions on Energy Conversion, 1993, 8(3), p. 576-583. 
[3] J. Hollingum. Robots Explore Underground Pipes. Industrial Robot-An International Journal, 1998, 25(5), p. 321-325.

[4] Z. Wang and H. Gu. A Bristle-Based Pipeline Robot for Ill-Constraint Pipes. IEEE/ASME Transactions on Mechatronics, 2008, 13(3), p. 383-392.

[5] H. Qi, X. Zhang, H. Chen, and J. Ye. Tracking and Localization System for Pipeline Robot. Mechatronics, 2009, 19(1), p. 76-84.

[6] M. Tavakoli, L. Marques, and A. T. de Almeida. Development of an Industrial Pipeline Inspection Robot. Industrial Robot-An International Journal, 2010, 37(3), p. 309-322.

[7] J. JR. Okamoto, J. C. Adamowski, M. S. G. Tsuzuki, et al. Autonomous System for Oil Pipelines Inspection. Mechatronics, 1999, 9(7), p. 731-743.

[8] J. Park, D. Hyun, W. H. Cho, et al. Normal-Force Control for an In-Pipe Robot According to the Inclination of Pipelines. IEEE Transactions on Industrial Electronics, 2011, 58(12), p. 5304-5310.

[9] M. Ciszewski, T. Buratowski, M. Giergiel, et al. Virtual Prototyping, Design and Analysis of an In-Pipe Inspection Mobile Robot. Journal of Theoretical and Applied Mechanics, 2014, 52(2), p. 417-429. 